\title{
Signs and Symptoms of Temporomandibular Joint Disorders Related to the Degree of Mouth Opening and Hearing Loss
}

Panagiotis Kitsoulis ${ }^{1 *}$, Aikaterini Marini ${ }^{2}$, Kalliopi lliou ${ }^{3}$, Vasiliki Galani ${ }^{4}$, Aristides Zimpis $^{5}$, Panagiotis Kanavaros ${ }^{6}$ and Georgios Paraskevas ${ }^{7}$

\begin{abstract}
Background: The temporomandibular joint is a unique bi-condylar joint involved in mastication and speech. Temporomandibular joint disorders (TMD) have a range of symptoms, including aural symptoms, and are present in approximately $75 \%$ of normal populations. The present study examined the relationship between signs and symptoms of TMD and mouth opening, gender, joint and aural symptoms, and hearing loss.

Methods: The study involved 464 healthy Greek university students (156 men and 308 women) with a mean age of 19.6 years. Age, gender and maximum mouth opening was recorded. Mouth opening was measured using Vernier calipers. An anamnestic questionnaire was used to stratify the subjects into four groups based on TMD severity. Aural symptoms and an audiogram were recorded for each subject too. Data were analyzed using multifactor ANOVA, chi-square, t-test, Mann-Whitney and Kruskal-Wallis tests.

Results: The overall incidence of TMD signs and symptoms was 73.3\%. The incidence and severity was greater in females than males ( $p$-value $0.0001<0.05$ ). The number of aural symptoms was associated to the TMD severity ( $p$-value $0.0001<0.05$ ) as well as maximum mouth opening (p-value $0.004<0.05$ ). Audiometry showed that moderate and severe TMD was associated with hearing loss of median and low tones respectively ( $p$-value $0.0001<0.05$ ). TMJ pain ( $p$-value $0.0001<0.05$ ), TMJ ankylosis ( $p$-value $0.0001<0.05$ ), bruxism ( $p$-value $0.0001<0.05$ ) and ear itching ( $p$-value $0.0001<0.05$ ) were also found to be statistically different between TMD and non-TMD subjects.

Conclusions: TMD signs and symptoms were more common and severe in females than males. TMD severity is correlated with the degree of mouth opening and the number of aural symptoms. The absence or presence of mild TMD are associated with normal audiograms while moderate and severe TMD are related to hearing loss in median and low tones respectively. Bruxism, joint ankylosis, joint pain and ear itching were more common in TMD than non-TMD patients.
\end{abstract}

Keywords: temporomandibular disorders mouth opening, aural symptoms, hearing loss, audiometry

\section{Background}

The temporomandibular joint is critical for normal mouth function, and thus plays a role in chewing, swallowing, speaking, oral health and nutrition. The temporomandibular joint is a synovial joint containing an articular disk which allows for hinge and sliding movements. The articular surfaces are covered by avascular

\footnotetext{
* Correspondence: pkitsoulis@hotmail.com

'Orthopaedic Surgeon, Professor of Anatomy-Histology-Embryology, Medical School, University of loannina, Greece

Full list of author information is available at the end of the article
}

and non-innervated fibrocartilage which has a high regenerative capacity. The temporalis and masseter muscles control joint's motion.

The term temporomandibular disorders (TMD) is a collective one, representing a sub-classification of muscle-skeletal disorders, and more specifically a number of signs and symptoms involving the masticatory muscles, the temporomandibular joint and associated structures. It is estimated that about one third of adults have TMD symptoms [1]. TMD has been related to stress, age, gender, personality and other systematic factors [2]. 
The maximum mouth opening distance is a generally accepted measurement to estimate temporomandibular joint mobility and function [3]. Mouth opening can be measured using gauges or calipers, and while the normal range differs between populations, the critical functional opening is $35-40 \mathrm{~mm}[4,5]$.

Aural symptoms such as tinnitus, otalgia, dizziness or vertigo, otic fullness sensation, hyperacousia or hypoacousia are thought to be associated with TMD [6,7], while their incidence reaches $85 \%$ in TMD patients [8]. A causative role of TMD in otic symptomatology remains a matter of debate. Several studies have shown that aural symptoms may have no otic origin [9]. Theories on the etiology of aural symptoms are based mainly on the common embryologic origin of the temporomandibular joint and the middle ear from Meckel cartilage, the presence of structures that connect the middle ear with the temporomandibular joint and the common innervation of the masticatory muscles and the ear.

The present study examined 464 healthy young Greek adults for the presence of TMD signs and symptoms. The study analyzed data for probable correlations between TMD severity and range of mouth opening, aural and joint symptoms and hearing loss. A feature of the study was the use of audiography to objectively assess the co-existence of hearing loss and TMD.

\section{Methods}

\section{Material}

The study was performed in the Laboratory of Anatomy at the Medical School of the University of Ioannina. The project involved 464 volunteers (156 males and 308 females) aged from 18 to 26 years (mean age 19.6 years) who attending the University of Ioannina. All volunteers were healthy, without active disease or craniofacial anomalies.

Maximum mouth opening $(\mathrm{mm})$ was measured using a $1 \mathrm{~mm}$ calibrated Vernier caliper. Subjects opened their mouths as wide as possible, and the inside jaws of the caliper were used to measure the interincisal distance (the distance between the edges of the upper and lower central incisors). During measurements, subjects were seated in a chair with head supported and held in the anatomical position in order to control for additional movements of the cervical, thoracic and lumbar spine [10]. Only the maximum active mouth opening was measured in order to avoid errors due to different applied force during passive opening [11]. Each measurement was repeated and assessed twice under the supervision of two observers.

At the completion of the measurement, the gender and age of each subject was recorded. Temporomandibular symptoms were assessed using a validated self-report anamnestic questionnaire composed of 10 questions [12].
The questionnaire asked about any history of difficulties in opening the mouth or moving the mandible sideways, discomfort while chewing, headaches, neck and shoulder pain, otalgia, temporomandibular joint sounds, abnormal biting, unilateral chewing and facial pain. We relied on the subject's sense of joint sounds, as referred elsewhere [13]. The possible questionnaire answers were "YES", "NO" and "SOMETIMES", and subjects were directed to choose only one of those answers for each question. Scores were obtained based on those answers. For each "YES" the score was "2", while for each "NO" or "SOMETIMES" the score was " 0 " and "1" respectively. For bilateral ear pain or temporomandibular joint sounds one more point was added and one more for intense headaches. Those scores were used to create four TMD groups: non- (score 0-3), mild (score 4-8), moderate (score 9-14) or severe (score 15-23) [12]. In addition to the questionnaire, subjects were asked about three further probable TMD related symptoms: bruxism, temporomandibular joint ankylosis and history of joint pain. Joint ankylosis was evaluated only if addressed to a physician for that reason.

Using another questionnaire, subjects were asked whether they had experienced the following aural symptoms: a feeling of fullness or pressure in ear, brief vertigo (less than 5 minutes), cold and warm air sensitivity, itching of the acoustic canal, and a history of diagnosed hearing loss, more than four events of otitis, tinnitus or ear pain. Subjects were categorized based on having none or less than two of those symptoms, more than two of the above symptoms, and more than four of the aforementioned symptoms.

After completing questionnaires, the volunteers were taken to a separate room where tympanometry and pure tone audiography were conducted to objectively evaluate hearing loss. Pure tone audiography included tones between $125-8000 \mathrm{~Hz}$. All subjects were found to be normal in terms of tympanometry results [14]. Each audiography pattern was then interpreted according to the Rösel calculator as a percentage of hearing loss for each ear [15]. Subjects were categorized according to five types of quantitative hearing loss: $\leq 15 \%$ in both ears, $>15 \%$ but $<20 \%$ in one ear, $>15 \%$ but $<20 \%$ in both ears, $\geq 20 \%$ in one ear and $\geq 20 \%$ in both ears. They were also categorized into five groups based on qualitative hearing loss according to audiological findings: no hearing loss, loss of low tones $(125-250 \mathrm{~Hz})$ in one and in both ears, unilateral loss of median tones $(25-4000 \mathrm{~Hz})$ and bilateral loss of median tones. No participant had a loss of high tones (4000-8000 $\mathrm{Hz}$ ) hearing, hence no such category was required.

All subjects gave written informed consent regarding participation and publication of the data. The study was approved by the Ethics Committee of the Medical School of University of Ioannina. 


\section{Statistical analysis}

Our variables for analysis were the age, the gender, the number of aural symptoms, range of mouth opening, and the degree of qualitative and quantitative hearing loss. ANOVA and t-tests were used to compare normally distributed variables, and Kruskal-Wallis and Mann-Whitney tests were used for comparing non-normally distributed variables (i.e. mouth opening in TMD and non-TMD subjects, mouth opening and gender, mouth opening and TMD severity). Chi-square test was used to compare both normally and non-normally distributed variables. We also investigated for correlations between TMD severity and bruxism, joint ankylosis, joint pain and ear itching using chi-square testing.

Analysis was performed on anonymous data using SPSS version 18. Kolmogorov-Smirnov and ShapiroWilk tests of normality were employed to determine data distribution. The level of statistical significance was $\mathrm{p}<0.05$. The confidence interval was $95 \%$.

\section{Results}

Of the 464 study subjects, $73.3 \%$ were diagnosed with TMD, and $26.7 \%$ were diagnosed as not having TMD. Of the 340 TMD subjects, TMD was classified as mild in $31.76 \%$, moderate in $40 \%$ and severe in $28.2 \%$. In addition, the incidence and severity of TMD was greater in women than men $(\mathrm{p}<0.05)$. The mean mouth opening was $46.56 \mathrm{~mm}$ in men and $44.43 \mathrm{~mm}$ in women ( $\mathrm{p}<$ 0.05 ; Mann-Whitney $U$ test). The range of mouth opening was greater in men than women (Tables 1, 2 and 3).

The mean mouth opening for the overall population was $45.08 \mathrm{~mm}$. The mean mouth opening was 46.45 $\mathrm{mm}$ in the non-TMD, and $44.59 \mathrm{~mm}$ in the TMD group ( $p<0.05$; Mann-Whitney $U$ test). Analysis of mouth opening distance in the four TMD groups showed that the mean mouth opening was $46.45 \mathrm{~mm}$ in the non-TMD group, in the mild TMD group 45.37 $\mathrm{mm}, 44.07$ and $44.43 \mathrm{~mm}$ in the moderate and severe TMD groups respectively. Kruskal -Wallis analysis indicated that mean mouth openings differed significantly between the TMD groups ( $\mathrm{p}<0.05$ ) (Tables 4,5 and 6).

Pain in the temporomandibular joint was reported by 96 subjects. It was experienced by $3 \%$ of the non-TMD group, $11.1 \%$ of the mild group, $8.8 \%$ of the moderate group and $70.8 \%$ of the severe TMD group. Chi-square test showed that temporomandibular joint pain was more likely to be present in the TMD than non-TMD patients
Table 2 Chi-square test reveals statistically significant relation of sex and TMD incidence and severity

\begin{tabular}{lccc}
\hline \multicolumn{4}{c}{ Chi-Square Tests } \\
\hline & Value & df & Asymp. Sig. (2-sided) \\
\hline Pearson Chi-Square & $26.309^{a}$ & 3 & .000 \\
\hline Likelihood Ratio & 26.739 & 3 & .000 \\
\hline Linear-by-Linear Association & 22.306 & 1 & .000 \\
\hline N of Valid Cases & 464 & & \\
\hline $\begin{array}{l}\text { a. } 0 \text { cells }(, 0 \%) \text { have expected count less than 5. The minimum expected } \\
\text { count is 32,28. }\end{array}$
\end{tabular}

$(\mathrm{p}<0.05)$ History of temporomandibular joint ankylosis was reported by 52 subjects. It was referred by $3.7 \%$ of mild, $2.9 \%$ of moderate and $45.8 \%$ of severe TMD subjects. No non-TMD participants had joint ankylosis. Chi-square test found that joint ankylosis was more likely to be found in TMD patient than non-TMD subjects $(\mathrm{p}<0.05)$. Bruxism was reported by 80 subjects. It was recorded in $0 \%$ of non-, $7.4 \%$ of mild, $29.4 \%$ of moderate, and $33.3 \%$ of severe TMD patients. Chi-square test showed that bruxism was more likely to occur in TMD than non-TMD subjects $(\mathrm{p}<0.05)$. Ear itching was reported by 92 subjects. It was experienced by $12.9 \%$ of non-, $11.1 \%$ of mild, $26.4 \%$ of moderate and $29.1 \%$ of severe TMD subjects. Chi-square test revealed that ear itching differed between TMD groups $(\mathrm{p}<0.05)$. Ear itching was commoner in TMD than non-TMD subjects (Tables 7, 8).

Subjects were assessed for their aural symptoms. We found that $62 \%$ had no or less than two symptoms, $28.44 \%$ had $>2$ symptoms, and $9.4 \%$ had $>4$ symptoms. We analyzed the number of aural symptoms in relation to TMD severity. The majority of the non-TMD (74.2\%) or mild TMD (70.4\%) subjects had no aural symptoms. For the moderate TMD group, 55.9\% had none and $38.2 \%$ had more than two aural symptoms. In the severe TMD group, $25 \%$ had more than two and $29.2 \%$ had more than four aural symptoms. Chi-square test showed that the severity of TMD is related to the number of aural symptoms. Qualitative assessment showed that $51.7 \%$ of the subjects had normal hearing, $13.8 \%$ and $18.1 \%$ of the subjects had unilateral and bilateral low tones loss respectively, and $8.6 \%$ and $7.8 \%$ of the subjects had bilateral and unilateral median tones loss respectively. We analyzed qualitative hearing loss and TMD groups using chi-square test. We found that TMD severity was correlated with hearing loss $(p<0.05)$.

Table 1 Presence of temporomandibular joint disorders and mouth opening distance in men and women

\begin{tabular}{|c|c|c|c|c|c|c|c|c|c|}
\hline & & & \multicolumn{4}{|c|}{ TMD group } & \multicolumn{3}{|c|}{ Mouth opening (mm) } \\
\hline & & & non & mild & moderate & severe & $\mathbf{N}$ & Mean & Std. Deviation \\
\hline \multirow[t]{2}{*}{ Gender } & male & Count & 56 & 48 & 32 & 20 & 156 & 46.5641 & 7.21931 \\
\hline & female & Count & 68 & 60 & 104 & 76 & 308 & 44.3377 & 5.82617 \\
\hline
\end{tabular}


Table 3 Mann-Whitney reveals a statistically significant difference of mouth opening between men and women

\begin{tabular}{lc}
\hline & Test Statistics $^{\text {a }}$ \\
\hline Mann-Whitney U & Mouth opening \\
\hline Wilcoxon W & 19240.000 \\
\hline$Z$ & 66826.000 \\
\hline Asymp. Sig. (2-tailed) & -3.512 \\
\hline
\end{tabular}

a. Grouping Variable: gender.

Most subjects without hearing loss belonged to the nonTMD group (46.6\%), while most subjects with low tones hearing loss in both ears belonged to the severe TMD group (47.6\%). For median tones, hearing loss in both ears was more common in moderate $(50 \%)$ compared to severe TMD (40\%) subjects. Furthermore, most mildTMD subjects had no hearing loss (59.3\%), Quantitative assessment using audiograms showed that $61.2 \%$ of the subjects had $\leq 15 \%$ hearing loss in both ears, $22.4 \%$ had $>15 \%$ loss in one ear, $5.2 \%$ had $>15 \%$ loss in both ears, $6.9 \%$ had $\geq 20 \%$ loss in one ear and $4.3 \%$ had $\geq 20 \%$ loss in both ears. Chi-square test showed that there was a relationship between TMD severity and degree of hearing loss ( $\mathrm{p}<0.05)$. Most non- and mild TMD subjects had $\leq 15 \%$ hearing loss. For the severe TMD group, 25\% had no hearing loss, $16.7 \%$ had > $15 \%$ loss in one ear and $16.7 \%$ had $>15 \%$ loss in both ears, $20.8 \%$ had $\geq 20 \%$ loss in one ear, and $20.8 \%(n=20)$ had $\geq 20 \%$ loss in both ears (Tables 9, 10).

\section{Discussion}

The present study examined the relationship between TMD symptoms and signs and mouth opening, gender, aural symptoms, temporomandibular joint pain, temporomandibular joint ankylosis, bruxism and hearing loss.

We found that $73.3 \%$ of the young Greek adult population examined in this study had TMD symptoms and signs. This finding is consistent with previous studies involving college students $[12,16]$. Compared to previous studies on young populations, the present population had fewer subjects with mild TMD (31.76\%) and more with moderate (40\%) and severe TMD (28.2\%).

Table 4 Mouth opening distance in non-TMD, TMD subjects and TMD subgroups. Descriptive statistics

\begin{tabular}{llccc}
\hline & TMD & N & Mean & Std. Deviation \\
\hline $\begin{array}{l}\text { Mouth opening } \\
(\mathrm{mm})\end{array}$ & Non-TMD & 124 & 46.4476 & 7.16257 \\
\hline & TMD & 340 & 44.5891 & 6.11675 \\
\hline & Mild & 108 & 45.3704 & 5.85908 \\
\hline & Moderate & 136 & 44.0792 & 5.69746 \\
\hline & Severe & 96 & 44.4346 & 6.93428 \\
\hline
\end{tabular}

Table 5 Mann- Whitney test shows a statistically significant difference of mouth opening between nonTMD and TMD subjects

\begin{tabular}{lc}
\hline \multicolumn{2}{c}{ Test Statistics $^{\text {a }}$} \\
\hline Mann-Whitney U & Mouth opening \\
\hline Wilcoxon W & 5361.000 \\
\hline$Z$ & 9894.000 \\
\hline Asymp. Sig. (2-tailed) & -2.874 \\
\hline a. Grouping Variable: TMD vs non-TMD. & 0.005 \\
\hline
\end{tabular}

We found that mouth opening differed, according to TMD severity, and the least opening was observed in the severe TMD group. Interestingly, we found that while mouth opening was less in severe TMD subjects $(44.43 \mathrm{~mm})$ compared to non-TMD subjects $(46.45$ $\mathrm{mm})$, it was greater in severe TMD subjects than moderate TMD ones $(44.08 \mathrm{~mm})$. It has already been reported that joint hypermobility is often present in TMD patients [2]. Mouth opening cannot be used as a sole criterion of TMD presence and the range of mouth opening found in our population could be considered as functional. Nevertheless, for the needs of our study, we compared the values found for each stratification group even of considered normal.

Ethnicity is believed to affect the degree of mouth opening [12,17]. The present study is the first focused on a young Greek adult population. The overall mean mouth opening recorded was $45.09 \mathrm{~mm}$. The only previous study of a Greek population took place in 1989 and involved participants aged between 18 and 70 years. The average maximum mouth opening was $52.85 \mathrm{~mm}$ for males and $48.34 \mathrm{~mm}$ for females [18]. Studies of other ethnicities found that the average mouth opening was $49.10 \mathrm{~mm}$ in Chinese (51.11 $\mathrm{mm}$ for those aged 20-30 years) [19], $49.8 \mathrm{~mm}$ in Americans aged 16-70 years [20], $47.1 \mathrm{~mm}$ in Nepalese population aged 18-68 years [21], $50.77 \mathrm{~mm}$ in French aged 18-84 years [22], and $55.9 \mathrm{~mm}$ in Swedes aged 18-25 years [23]. It ranged between 41-43 $\mathrm{mm}$ in Irish [24] and 35-61 $\mathrm{mm}$ in Croatians [25].

We found that TMD was more prevalent and severe in women than men. This observation is consistent with

Table 6 Kruskal-Wallis reveals a statistically significant difference of mouth opening among TMD groups

\begin{tabular}{lc}
\hline & \multicolumn{2}{c}{ Test Statistics ${ }^{\mathbf{a}, \mathbf{b}}$} \\
\hline Chi-square & Mouth opening \\
\hline $\mathrm{D}$ & 12.368 \\
\hline Asymp. Sig. & 3 \\
\hline a. Kruskal Wallis Test. & .004 \\
b. Grouping Variable: TMD.
\end{tabular}


Table 7 Presence of temporomandibular joint ankylosis, bruxism, aural symptoms, pain and ear itching according to TMD group

TMJ pain *,ankylosis,bruxism,aural symptoms,pain TMD Crosstabulation

\begin{tabular}{lllllllll}
\hline \multirow{2}{*}{ Ankylosis } & & & \multicolumn{5}{c}{ TMD group } \\
\cline { 2 - 8 } & & & non & mild & moderate & severe & Total \\
\cline { 2 - 8 } & Yes & Count & 124 & 104 & 132 & 52 & 412 \\
\hline Bruxism & No & Count & 0 & 4 & 4 & 44 & 52 \\
\cline { 2 - 8 } & Yes & Count & 0 & 8 & 40 & 32 & 80 \\
\hline Aural & $0-2$ aural & Count & 92 & 76 & 76 & 44 & 288 \\
Symptoms & symptoms & & & & & & \\
\cline { 2 - 8 } & $>2$ aural & Count & 28 & 28 & 52 & 24 & 132 \\
\cline { 2 - 8 } & symptoms & & & & & & \\
\cline { 2 - 8 } & symptoms & Count & 4 & 4 & 8 & 28 & 44 \\
\hline Pain & No & Count & 120 & 96 & 124 & 28 & 368 \\
\cline { 2 - 8 } & Yes & Count & 4 & 12 & 12 & 68 & 96 \\
\hline Ear itching & No & Count & 108 & 96 & 100 & 68 & 372 \\
\cline { 2 - 8 } & Yes & Count & 16 & 12 & 36 & 28 & 92 \\
\hline
\end{tabular}

previous studies, as it has been reported that women present an increased risk of 1.5-2 or 2-9 times compared to men to develop TMD [17].

The current study found that pain in the temporomandibular joint was the most common symptom (27.05\%) of TMD as reported elsewhere [2]. Temporomandibular joint pain is caused by the pathological contraction of the masticatory muscles which stimulates extravascular production of inflammation associated substances around the temporomandibular joint [26]. The incidence of pain in the present study is in accordance with the theory that TMD is more prevalent in early adulthood [27].

Joint sounds are regarded as the commonest sign of TMD (13.5\%) [28], and are more frequent and severe in older populations. Joint sounds, such as clicks and friction, indicate temporomandibular joint derangement or joint disk displacements.

We found that bruxism was present in $27.02 \%$ of those with TMD and in none of the non-TMD subjects.

Table 8 Chi-square tests show a statistically significant relation of TMJ ankylosis, bruxism, number of aural symptoms, pain, ear itching and TMD severity

\begin{tabular}{ll}
\hline & Chi-Square Tests \\
\hline TMJ symptom/sign & p-value \\
\hline Ankylosis & 0.000 \\
\hline Bruxism & 0.000 \\
\hline Aural Symptoms & 0.000 \\
\hline Pain & 0.000 \\
\hline Ear Itching & 0.000 \\
\hline
\end{tabular}

Others reported that bruxism was present in 7.4\%-27.2\% of TMD subjects [16]. Bruxism, as clenching or grinding, may be a source or a perpetuating factor for TMD. Some investigators reported that non-TMD and TMD patients have different electromyographic patterns [29] while others failed to find a difference [30].

Head and neck ache may be of neurological, vascular, muscular, ligamental or bony origin. The temporomandibular joint has muscular and ligamentary connections to the cervical region forming a functional complex. Headache is regarded as the most common symptom (22\%) of TMD patients [16], while $55 \%$ of chronic headache patients referred to a neurologist had signs or symptoms of TMD [31]. The muscles of the neck and trunk are reported to have a greater electromyographic activity in TMD subjects [32] sensitizing the sympathetic nerves of the autonomous nervous system and leading to headache via the trigeminal nerve.

We found that TMD severity is correlated with the number of aural symptoms. Costen, in 1934, firstly associated aural and craniosinusal symptoms with TMD, and named it Costen's or Otognathic Syndrome [9]. There are four main theories concerning the co-existence of aural and temporomandibular joint dysfunction symptoms. The first one suggests that disposition of the joint disk during jaw movement increases pressure in the Eustachian tube, the ear structures, the auriculotemporal and masseteric nerve and some branches of the deep posterior temporal nerve. The auriculotemporal nerve innervates the TMJ, the tympanic membrane, the anterosuperior part of the external ear and the tragus, and its stimulation gives the sense of otalgia [8]. We observed that $10.8 \%$ of TMD subjects reported ear pain, and that $75 \%$ of those TMD subjects had severe TMD. A number of series report ear pain in 35\% of TMD patients [28], while $70 \%$ of temporomandibular joint pain cases are described as otalgia particularly during acute or subacute inflammation of the temporomandibular joint [33]. Additionally, we found that the rarely investigated symptom of ear itching was more common in TMD than nonTMD subjects, as reported elsewhere [28].

The second theory involves the tiny ligament called the discomalleolar or Pinto's ligament, which originates from the anterior malleolar process, passes through the petrotympanic fissure and inserts in the medial and posterosuperior part of the articular capsule and the disk $[34,35]$. In displacement of the temporomandibular joint disk, the discomalleolar ligament can lead to traction of the malleus [36], the ossicular chain and the tympanic membrane [34]. Furthermore, in disk displacement, the intralaminal vascular tissue moves forward and is trapped between the head of the condyle and the roof of the fossa, leading to oedema, leakage and fibrosis [37]. The petrotympanic fissure plays an important role in 
Table 9 Quantitative hearing loss and loss of tonal hearing according to TMD group

\begin{tabular}{|c|c|c|c|c|c|c|c|}
\hline \multicolumn{8}{|c|}{ Tonal hearing loss * TMD Crosstabulation } \\
\hline & & & \multicolumn{4}{|c|}{ TMD Group } & \multirow[b]{2}{*}{ Total } \\
\hline & & & Non & mild & moderate & severe & \\
\hline \multirow[t]{9}{*}{ Hearing loss } & normal $\leq 15 \%$ & Count & 104 & 88 & 68 & 24 & 284 \\
\hline & In one & Count & 16 & 16 & 56 & 16 & 104 \\
\hline & ear $>15 \%$ & & & & & & \\
\hline & In one & Count & 4 & 0 & 8 & 20 & 32 \\
\hline & ear $\geq 20 \%$ & & & & & & \\
\hline & In both & Count & 0 & 4 & 4 & 16 & 24 \\
\hline & ears $>15 \%$ & & & & & & \\
\hline & In both & Count & 0 & 0 & 0 & 20 & 20 \\
\hline & ears $\geq 20 \%$ & & & & & & \\
\hline \multirow[t]{5}{*}{ Tonal hearing loss } & No loss & Count & 112 & 64 & 52 & 12 & 240 \\
\hline & Loss in low tones in both ears & Count & 0 & 12 & 32 & 40 & 84 \\
\hline & Loss in low tones in one ear & Count & 8 & 24 & 8 & 24 & 64 \\
\hline & Loss in median tones in both ears & Count & 0 & 4 & 20 & 16 & 40 \\
\hline & Loss in median tones in one ear & Count & 4 & 4 & 24 & 4 & 36 \\
\hline
\end{tabular}

Tonal hearing loss, ear itching, hearing loss -TMD.

hosting the anterior malleolar ligament, the anterior tympanic artery and the chorda tympani nerve. Additionally, the petrotympanic fissure brings in contact the temporomandibular joint with the middle ear. Thus, beginning from the joint capsule, inflammation can spread to the origin of the levator and the tensor palatini muscles, and finally to the cul-de-sac over the vulnerable isthmus of the Eustachian tube, causing obstruction of the tube. A closed Eustachian tube may be responsible for the feeling of ear fullness, otalgia and serous otitis media [38]. This fissure's length and position may influence the development of otalgia, tinnitus, hearing loss and vertigo [35].

The third theory proposes that common innervation of the tensor veli palatini, tensor tympani, masseter, temporalis and pterygoid muscles by the motor nucleus of the trigeminal nerve is the underlying cause of the aural symptomatology in TMD patients [36]. Tensor tympani, tensor veli palatini and stapedial muscles are called auditory muscles, while the tensor veli palatini and the tensor tympani muscles are also called accessory mastication muscles [7]. Strong sounds cause stapedial muscle contraction, which improves auditory

Table 10 Statistically significant relation of tones hearing loss, quantitative hearing loss and TMD via chi-square tests

\begin{tabular}{lc}
\hline \multicolumn{2}{c}{ Chi-Square Tests } \\
\hline \multicolumn{2}{c}{ p-value } \\
\hline Quantitative hearing loss & 0.000 \\
\hline Tonal hearing loss & 0.000 \\
\hline
\end{tabular}

discrimination [39], while tensor tympani muscle plays a role in the discrimination of low tones. Tensor tympani muscle also contracts in strong sounds, protecting from sound trauma, and vocalization, chewing, swallowing and facial muscle contraction [40]. Malcontraction of the tensor tympani muscle pulls the ossicular chain medially in the middle ear and thus may alter the aural conductive system [9] causing hyperacousia or hypoacousia even with normal audiometric values [41]. It has also been shown that the tensor tympani muscle is dysfunctional in TMD patients, leading to subjective hearing loss [42]. The tensor tympani and stapedial are antagonistic muscles, and with the tympanic membrane are responsible for the appropriate balance and function of the ossicular chain in the middle ear (malleus, incus and stapes). If the tensor tympani or stapedial contract inappropriately, then the perilymphatic and endolymphatic pressures in the inner ear are changed via the oval window, causing vestibular and cochlear impulse imbalance [43]. Changes in endolymph pressure affect the hair cells of the inner ear, which also results in aural symptoms. Electromyographical studies show that the tensor tympani and tensor and tensor veli palatini work simultaneously during swallowing, leading to ventilation of the Eustachian tube [41] raising the intratympanic pressure. The Eustachian tube is actively opened by the tensor palatini and passively opened by the levator palatini muscle. In TMD, the tensor tympani muscle is hypertonic, impeding this normal mechanism. Such a pathological state may lead to hypoacusia, tinnitius, vertigo, otalgia, otic fullness sensation and otitis media $[9,44]$. Additionally, TMD patients cannot open their 
mouths wide to yawn, which leads to poor Eustachian tube function as well [36]. One study reported that ear fullness was experienced by $13.5 \%$ of TMD and $4.7 \%$ of non-TMD patients $[R R=2.87]$, while in other series involving older populations the relative risk ratio was 14.0 [28]. Objective tinnitus is caused by the palatine and middle ear myoclonus producing rhythmic movement of the tympanic membrane to the tension of the stapedial and tensor tympani muscle. Tinnitus related to TMD is thought to be produced by the lateral pterygoid muscles and the discomalleolar ligament [36].

Furthermore, every movement of the neck or jaw exerts tension on the carotid sheath, increasing the endolymphatic pressure of the hair cells in the cochlea via impedance of the saccus endolymphaticus' pressureregulating mechanism, causing tinnitus and vertigo [37]. In bruxism and TMD, the tensor veli palatini dysfunctions and may change the position of tympanic membrane and malleus due to its anatomic association with the tensor tympani [45]. Thus, the velopharyngeal, neck and face movements play a major role in otic-TMD symptomatology [7].

The results of a previous study suggested that TMD peripherally sensitizes the V and VII pairs, leading to tonic spasm of these middle ear muscles. This process may cause low tones hearing loss. We found that severe TMD was associated with low tones hearing loss, while moderate TMD correlated with median tones hearing loss. Furthermore, the degree of hearing loss increased with TMD severity. In a study of 44 TMD patients, ear fullness was recorded in $13.6 \%$, vertigo in $13.6 \%$ and hearing loss in $6.8 \%$ of the subjects. In that same study, audiometry revealed a sensorineural hearing loss in only one subject, at 28-30 dB [46]. Other studies reported no difference in audiographic findings between TMD and non-TMD patients [47], and that hearing loss was not correlated to TMD severity [48]. It has also been previously reported that TMD symptoms are more common in subjects who present with sudden sensorineural hearing loss [49].

Finally, the fourth theory proposes that psychosocial disorders are responsible for the co-existence of aural symptoms in TMD patients [50].

The limitation of our study is the absence of clinical diagnosis of TMD. No clinical examination or other laboratory findings confirmed or rejected each participant's score according to his anamnestic questionnaire. The tool employed only describes signs and symptoms of TMD which may not be seen as a synonym of TMD presence. Thus, many volunteers of this study could not be diagnosed as TMD patients. For the convenience of the results' interpretation we stratified our subjects in four TMD severity groups referring to the number of TMD signs and symptoms. Nevertheless, the results of several studies based on such data verify the utility of evaluation via only an anamnestic questionnaire $[12,28]$.

\section{Conclusions}

The present study found that TMD severity was associated with gender (more common and severe in women), range of mouth opening, the number of aural symptoms, ear itching, temporomandibular joint ankylosis, temporomandibular joint pain, bruxism and qualitative and quantitative hearing loss. The close embryological, anatomical and functional relationships between the temporomandibular joint and the middle ear may explain the link between aural symptoms and TMD severity. A feature of the present study was the use of audiometry to assess hearing loss in TMD. Finally, this is the first study to measure mouth opening in young Greek adults.

\section{Acknowledgements}

We thank Mr. Tsiolis Michalis for performing the audiograms, interpreting the data, and consenting to their publication in the preset study.

\section{Author details}

${ }^{1}$ Orthopaedic Surgeon, Professor of Anatomy-Histology-Embryology, Medical School, University of Ioannina, Greece. ${ }^{2}$ Intern of Internal Medicine, Assistant in the Laboratory of Anatomy-Histology-Embryology, Medical School, University of Ioannina, Greece. ${ }^{3}$ Assistant in the Laboratory of AnatomyHistology-Embryology, Medical School, University of loannina, Greece. ${ }^{4}$ Associate professor of Anatomy-Histology-Embryology, Medical School, University of loannina, Greece. ${ }^{5}$ Orthopaedic Surgeon, Lecturer of Anatomy, Medical School, University of Larissa, Thessaly, Greece. ${ }^{6}$ Professor and Chairman of Anatomy-Histology-Embryology, Medical School, University of Ioannina, Greece. ${ }^{7}$ Orthopaedic Surgeon, Assistant Professor of Anatomy, Medical School, Aristotle University, Thessaloniki, Greece.

\section{Authors' contributions}

PK conceived of the study and participated in its design and coordination. AM and VG carried out the measurements and drafted the manuscript. KI carried out the statistical analysis of the study. AZ, PK and GP participated in the interpretation of the data and in the design and coordination of the study. All authors read and approved the final version of the manuscript.

\section{Competing interests}

The authors declare that they have no competing interests.

Received: 7 September 2010 Accepted: 25 May 2011

Published: 25 May 2011

\section{References}

1. JJ Buescher, Temporomandibular joint disorders. Am Fam Physician. 76, 1477-82 (2007)

2. K Oral, B Bal, B Ebeoglu, S Dincer, Etiology of temporomandibular disorder pain. Agri. 21(3):89-94 (2009)

3. N Walker, RW Bohannon, D Cameron, Discriminant validity of temporomandibular joint range of motion measurements obtained with a ruler. J Orthop Sports Phys Ther. 30(8):484-92 (2000)

4. B Scott, C Butterworth, D Lowe, SN Rogers, Factors associated with restricted mouth opening and its relationship to health-related quality of life in patients attending a Maxillofacial Oncology clinic. Oral Oncol. 44, 430-438 (2008). doi:10.1016/j.oraloncology.2007.06.015

5. SF Dworkin, L LeResche, Research diagnostic criteria for temporomandibular disorders: review, criteria, examinations and specifications, critique. J Craniomandib Disord. 6(4):301-55 (1992)

6. EF Wright, Otologic symptom improvement through TMD therapy. Quintessence Int. 38(9):e564-71 (2007) 
7. LM Ramirez, LE Ballesteros, GP Sandoval, Tensor tympani muscle: strange chewing muscle. Med Oral Patol Oral Cir Bucal. 12, E96-100 (2007)

8. G Salvetti, D Manfredini, S Barsotti, M Bosco, Otologic symptoms in temporomandibular disorders patients: is there evidence of an associationrelationship? Minerva Stomatol. 55, 627-37 (2006)

9. H Myrhaug, The incidence of the ear symptoms in cases of malocclusion and temporomandibular joint disturbances. Br J Oral Maxillofac Surg. 2, 28-32 (1964)

10. M Naeije, Local kinematic and anthropometric factors related to the maximum mouth opening in healthy individuals. J Oral Rehab. 29, 534-539 (2002). doi:10.1046/j.1365-2842.2002.00895.x

11. PU Dijkstra, LGM Bont, B Stegenga, G Boering, Angle of mouth opening measurement: reliability of a technique for temporomandibular joint mobility assessment. J Oral Rehab. 22, 263-268 (1995). doi:10.1111/j.13652842.1995.tb00084.x

12. DM Fonseca, Disfuncao craniomandibular (DCM) - diagnostico pela anamnese. FOB - Faculdade de Odontologia de Bauru. 1-116 (1992)

13. LM Gallo, R Airoldi, B Ernst, S Palla, Power spectral analysis of temporomandibular joint sounds in asymptomatic subjects. J Dent Res. 72(5):871-5 (1993). doi:10.1177/00220345930720050701

14. A Skevas, Otorhinolaryngology. (loannina, 2002), Third, pp. 102-106

15. HG Boenninghaus, D Röser, On the practical use of hearing loss tables and the establishment of decrease of earning capacity. Arch Ohren NasenKehlkopfheilkd. 175, 396-401 (1959). doi:10.1007/BF02103342

16. RM Feteih, Signs and symptoms of temporomandibular disorders and oral parafunctions in urban Saudi Arabian adolescents: a research report. Head Face Med. 2, 25 (2006). doi:10.1186/1746-160X-2-25

17. AS de Oliveira, EM Dias, RG Contato, F Berzin, Prevalence study of signs and symptoms of temporomandibular disorder in Brazilian college students. Braz Oral Res. 20(1):3-7 (2006)

18. M Mezitis, G Rallis, N Zachariades, The normal range of mouth opening. J Oral Maxillofac Surg. 47, 1028-9 (1989). doi:10.1016/0278-2391(89)90174-2

19. KT Yao, CC Lin, CH Hung, Maximum mouth opening of ethnic Chinese in Taiwan. J Dent Sci. 4(1):40-44 (2009). doi:10.1016/S1991-7902(09)60007-6

20. IM Sheppard, SM Sheppard, Maximal incisal opening: a diagnostic index? J Dent Med. 20, 13-15 (1965)

21. SC Cox, DM Walker, Establishing a normal range for mouth opening: its use in screening for oral submucous fibrosis. Br J Oral Maxillofac Surg. 35, 40-2 (1997). doi:10.1016/S0266-4356(97)90007-3

22. G Placko, V Bellot-Samson, S Brunet, L Guyot, O Richard, F Cheynet, C Chossegros, M Ouaknine, Normal mouth opening in the adult French polulation. Rev Stomatol Chir Maxillofac. 106, 267-71 (2005). doi:10.1016/ S0035-1768(05)86038-3

23. G Ageberg, Maximal mandibular movements in young men and women. Sven Tandlak Tidskr. 67, 81-100 (1974)

24. C Gallagher, V Gallagher, H Whelton, M Cronin, The normal range of mouth opening in an Irish population. J Oral Rehab. 31, 110-6 (2004). doi:10.1046/ j.0305-182X.2003.01209.x

25. R Celic, V Jerolimov, D Knezovic, Relationship of slightly limited mandibular movements to temporomandibular disorders. Braz Dent J. 15(2):151-154 (2004). doi:10.1590/S0103-64402004000200012

26. K Kaneyama, N Segami, M Nishimura, T Suzuki, J Sato, Importance of proinflammatory cytokines in synovial fluid from 121 joints with temporomandibular disorders. Br J Oral Maxillofac Surg. 40(5):418-23 (2002)

27. SF Dworkin, KH Huggins, LR LeResche, M Von Korff, J Howard, E Truelove, E Sommers, Epidemiology of signs and symptoms in temporomandibular disorders: I, Clinical signs in cases and controls. J Am Dent Assoc. 120, 273-81 (1990)

28. KW Cox, Temporomandibular disorder and new oral symptoms. Arch Otolaryngol Head Neck Surg. 134(4):389-393 (2008). doi:10.1001/ archotol.134.4.389

29. I Ardizone, A Celemin, F Aneiros, J Rio, T Sanchez, I Moreno, Electromyographic study of activity of the masseter and anterior temporalis muscles in patients with temporomandibular joint (TMJ) dysfunction: comparison with the clinical dysfunction index. Med Oral Patol Oral Cir Bucal. 15(1):e14-9 (2010)

30. MT John, H Frank, F Lobbezoo, M Drangsholt, KE Dette, No association between incisal tooth wear and temporomandibular disorders. J Prosthet Dent. 87, 197-203 (2002). doi:10.1067/mpr.2002.121167
31. EF Wright, EG Clark, ED Paunovich, RG Hart, Headache improvement through TMD stabilization appliance and self-management therapies. Cranio. 24, 104-11 (2006)

32. S Tecco, S Tete, M D'Attilio, L Perillo, F Festa, Surface electromyographic patterns of masticatory, neck, and trunk muscles in temporomandibular joint dysfunction patients undergoing anterior repositioning splint therapy. Europ J Orthod. 30, 592-597 (2008). doi:10.1093/ejo/cjn052

33. B Rubinstein, A Axelsson, GE Carlsson, Prevalence of signs and symptoms of craniomandibular disorders in tinnitus patients. J Craniomandib Dis Facial Oral Pain. 4, 186-92 (1990)

34. OF Pinto, A new structure related to the temporomandibular joint and middle ear. J Prosthet Dent. 12, 95-103 (1962). doi:10.1016/0022-3913(62) 90014-8

35. YF Ren, A Isberg, Tinnitus in patients with temporomandibular joint internal derangement. Cranio. 13, 75-80 (1995)

36. MM Ash, CM Ash, JL Ash, GM Ash, Current concepts of the relationship and management of temporomandibular disorders and auditory symproms. J Mich Dent Assoc. 72(11-12):550-555 (1990)

37. L Myers, Possible inflammatory pathways relating temporomandibular joint dysfunction to otic symptoms. J Craniomandib Pract. 6(1):65-70 (1988)

38. FK Marasa, BD Ham, Case reports involving the treatment of children with chronic otitis media with effusion via craniomandibular methods. J Craniomandib Pract. 6, 256-70 (1988)

39. I Klockhoff, $H$ Anderson, Reflex activity in the tensor tympani muscle recorded in man; preliminary report. Acta Otolaryngol. 51, 184-8 (1960). doi:10.3109/00016486009124480

40. LP Gray, The relationship between the "superior constrictor swallow" clicking of the ears and ear disease. J Laryngol Otol. 97, 1121-8 (1983)

41. J Schames, M Schames, E King, S Ulansey, J Boyd, E Schames, Trigeminal pharyngioplasty: treatment of the forgotten accessory muscles of mastication which are associated with orofacial pain and ear symptomatology. AJPM. 12, 102-12 (2002)

42. M Ogutcen-Toller, RP Juniper, Audiological evaluation of the aural symptoms in temporomandibular joint dysfunction. J Craniomaxillofac Surg. 21, 2-8 (1993)

43. HH Shapiro, RC Truex, The temporomandibular joint and the auditory function. J Am Dent Assoc. 30, 1147-68 (1943)

44. DP Malkin, The role of TMD dysfunction in the etiology of middle ear diseases. Int J Orthod. 25, 20-1 (1987)

45. AC Kierner, R Mayer, K $\vee$ Kirschhofer, Do the tensor tympani and tensor veli palatini muscles of man form a functional unit? A histochemical investigation of their putative connections. Hear Res. 165, 48-52 (2002). doi:10.1016/S0378-5955(01)00419-1

46. I Kaygusuz, T Karlidag, E Keles, S Yalcin, M Yildiz, HC Alpay, Ear symptoms accompanying temporomandibular joint diseases. Kulak Burum Bogaz Ihtis Derg. 16(5):205-8 (2006)

47. MO Toller, RP Juniper, Audiological evaluation of the aural symptoms in temporomandibular joint dysfunction. J Craniomaxillofac Surg. 21(1):2-8 (1993)

48. R Ciancaglini, P Loreti, G Radaelli, Ear, nose and throat symptoms in patients with TMD: the association of symptoms according to severity of arthropathy. J Orofac Pain. 8, 293-7 (1994)

49. R Axelsson, M Tullberg, M Ernberg, B Hedenberg-Magnusson, Symptoms and signs of temporomandibular disorders in patients with sudden sensorineural hearing loss. Swed Dent J. 33(3):115-23 (2009)

50. S Kuttila, M Kuttila, Y Le Bell, P Alanen, S Jouko, Aural symptoms and signs of temporomandibular disorder in association with treatment need and visits to a physician. Laryngoscope. 109, 1669-73 (1999). doi:10.1097/ 00005537-199910000-00022

\section{Pre-publication history}

The pre-publication history for this paper can be accessed here: http://www.biomedcentral.com/1472-6815/11/5/prepub

doi:10.1186/1472-6815-11-5

Cite this article as: Kitsoulis et al:: Signs and Symptoms of

Temporomandibular Joint Disorders Related to the Degree of Mouth Opening and Hearing Loss. BMC Ear, Nose and Throat Disorders 2011 11:5. 